\title{
Deep Reactive Ion Etching (DRIE) of High Aspect Ratio SiC Microstructures using a Time-Multiplexed Etch-Passivate Process
}

\author{
Laura J. Evans and Glenn M. Beheim \\ NASA Glenn Research Center, MS 77-1, 21000 Brookpark Rd., Cleveland OH 44135, USA \\ e-mail: Laura.J.Evans@nasa.gov, Glenn.M.Beheim@nasa.gov
}

Keywords: deep reactive ion etching (DRIE), MEMS, bulk micromachining, aspect ratio

\begin{abstract}
High aspect ratio silicon carbide (SiC) microstructures are needed for microengines and other harsh environment micro-electro-mechanical systems (MEMS). Previously, deep reactive ion etching (DRIE) of low aspect ratio $(\mathrm{AR} \leq 1)$ deep $(>100 \mu \mathrm{m})$ trenches in $\mathrm{SiC}$ has been reported. However, existing DRIE processes for SiC are not well-suited for definition of high aspect ratio features because such simple etch-only processes provide insufficient control over sidewall roughness and slope. Therefore, we have investigated the use of a time-multiplexed etch-passivate (TMEP) process, which alternates etching with polymer passivation of the etch sidewalls. An optimized TMEP process was used to etch high aspect ratio $(A R>5)$ deep $(>100 \mu \mathrm{m})$ trenches in $6 \mathrm{H}-\mathrm{SiC}$. Power MEMS structures (micro turbine blades) in $6 \mathrm{H}-\mathrm{SiC}$ were also fabricated.
\end{abstract}

\section{Introduction}

Microengines and other power MEMS frequently utilize silicon ( $\mathrm{Si}$ ) because the required high aspect ratio microstructures can be readily fabricated in Si using a time-multiplexed etch-passivate (TMEP) process (or Bosch process) [1,2]. Silicon, however, undergoes creep at relatively low temperatures, which limits operating temperatures and microengine power. Silicon carbide has superior mechanical properties at elevated temperatures, but micromachining methods for SiC are not yet well-developed. We investigated the TMEP process for the fabrication of high aspect ratio $\mathrm{SiC}$ microstructures because of the limitations of existing etch-only DRIE processes for SiC. An inductively coupled plasma (ICP) etch-only process using $\mathrm{SF}_{6}$ and $\mathrm{Ar}$ has been used at NASA Glenn to etch low aspect ratio structures to depths of several hundred $\mu \mathrm{m}$. This process, which is described fully in [3], has the advantage of providing residue-free deep etches, but is not suitable for high aspect ratio features because it produces rough sidewalls and a re-entrant profile. Fluorine plasma etching of $\mathrm{SiC}$ is inherently anisotropic, because ion bombardment is required to achieve an appreciable etch rate. However, the etch rate in the absence of ion bombardment is sufficient to cause roughening of the sidewalls when deep etching. To protect the sidewalls, we have adopted a modified TMEP process, which alternates etching with conformal deposition of an etch-inhibiting polymer. The polymer is quickly removed from the horizontal surfaces at the beginning of each ionassisted etch step but is longer-lived on the sidewalls. In addition, the TMEP process can provide improved control over the sidewall slope by decoupling etching and passivation, the relative balance of which determines the slope.

\section{Experimental}

The TMEP experiments were performed in a STS Multiplex ICP etcher, using $\mathrm{SF}_{6}$ for etching and $\mathrm{C}_{4} \mathrm{~F}_{8}$ for passivation. The samples were $\mathrm{n}$-type, off-axis, $\mathrm{Si}$-face $6 \mathrm{H}-\mathrm{SiC}$, with a resistivity of 0.065 $\Omega$-cm. An etch mask patterned with various width trenches was produced by selective electroplating of Ni to a thickness of approximately $5 \mu \mathrm{m}$. Design of experiments (DOE) was used to optimize the TMEP process for maximum etch rate, sidewall slope, and smoothness. The study was focused on three parameters known to strongly influence the etch characteristics of interest: platen power during the etch step, pressure (which was the same during both steps to enhance stability), and duration of the passivation step [1]. Coil power was fixed at the maximum generator 
output, $1000 \mathrm{~W}$, to maximize the plasma density. The duration of the etch step was fixed at $30 \mathrm{~s}$, the maximum value permitted by the ICP control software, to obtain appreciable etching of SiC during each step. Etches were performed using low and high values of etch-step platen power (50 and 100 $\mathrm{W})$, pressure (7 and $17 \mathrm{mT})$, and passivation-step duration (9 and $17 \mathrm{~s})$. The $2^{3}$ full factorial design was augmented with five repetitions of center point conditions $(75 \mathrm{~W}, 12 \mathrm{mT}, 13 \mathrm{~s})$ to provide a measure of process stability and variability. To enable automated handling by the ICP, the SiC samples ( 8 were fabricated from each $50-\mathrm{mm}$ wafer) were mounted on $100-\mathrm{mm}$ Si carrier wafers using photoresist as an adhesive. Prior to each etch, the sample surface was cleaned using a 10-min Ar sputter etch, then removed from the chamber while the chamber walls were conditioned by depositing polymer for $3 \mathrm{~min}$. Etch time was $10 \mathrm{hrs}$ to provide etch depths on the order of $100 \mu \mathrm{m}$. Etch depth was measured using surface profilometry and optical microscopy, and sidewall slope was measured via optical microscopy of cross-sectioned etch specimens.

\section{Results and Discussion}

SiC Etch Rate. Etch rate was found to be most strongly influenced by platen power and passivation time (etch rate was increased by decreasing passivation time or increasing platen power), while pressure was found to have a lesser effect. Figure 1 shows the $\mathrm{SiC}$ etch rate as a function of platen power and pressure, for a 9-s passivation time. The peak etch rate of $0.24 \mu \mathrm{m} / \mathrm{min}$ was obtained at 100-W platen power and 7-mT pressure. At this power and passivation time, increasing pressure to $17 \mathrm{mT}$ reduced the etch rate slightly, from 0.24 to $0.22 \mu \mathrm{m} / \mathrm{min}$. For comparison, the rate when etching at $100-\mathrm{W}$ platen power and $17-\mathrm{mT}$ pressure was $0.39 \mu \mathrm{m} / \mathrm{min}$ when no passivation step was used. Increasing the passivation time from 9 to $17 \mathrm{~s}$, at $100-\mathrm{W}$ platen power and $7-\mathrm{mT}$ pressure, reduced the etch rate by $29 \%$, to $0.17 \mu \mathrm{m} / \mathrm{min}$. This etch rate reduction can be largely attributed to the $21 \%$ reduction in the proportion of process time devoted to etching.
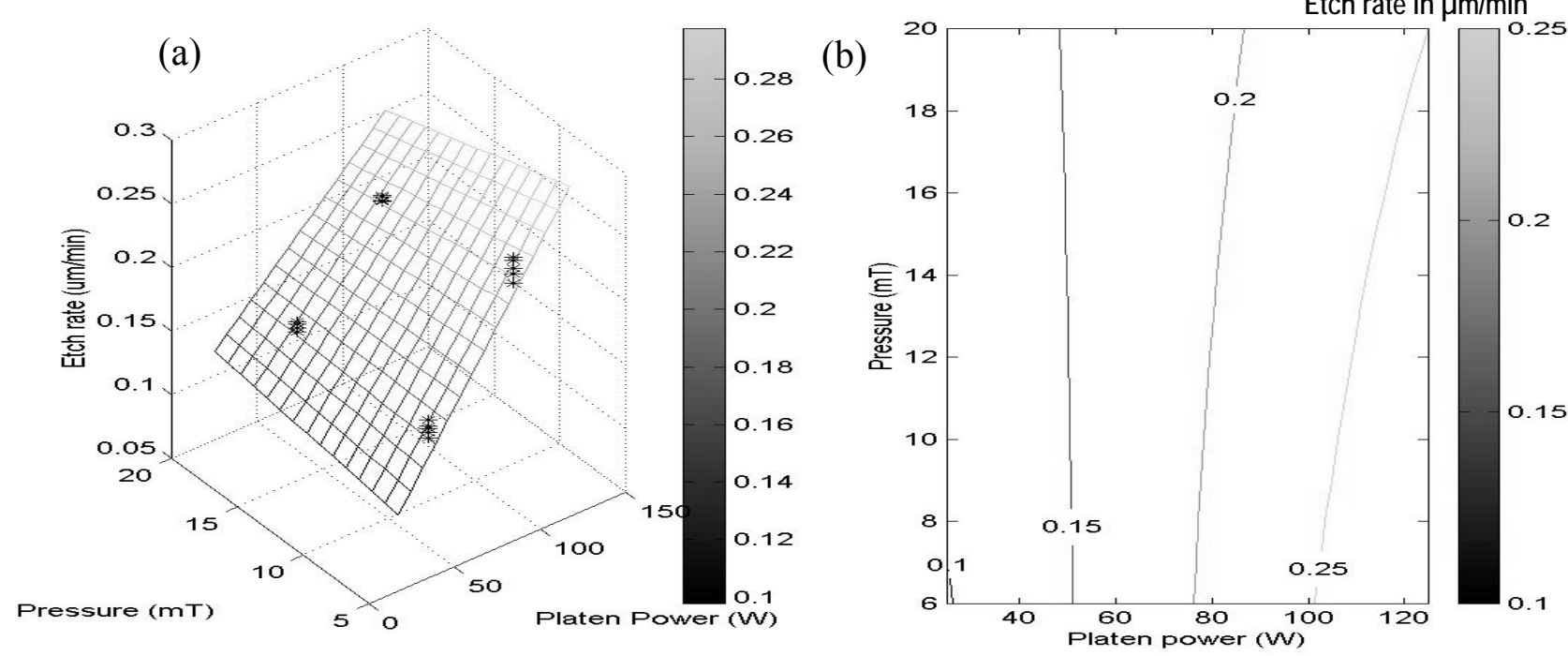

Fig. 1 - SiC etch rate as a function of platen power and pressure with a 9-s passivation time:

(a) response surface and experimental data points; (b) contour plot of response surface.

While an increase in platen power increases the etch rate (by increasing the energy with which ions bombard the SiC surface), it has a negative impact on selectivity, which determines the etch depth that can be achieved for a given mask thickness (as noted in the next section, platen power also has a significant impact on anisotropy). In a separate experiment, using 200 -nm-thick evaporated $\mathrm{Ni}$ masks, the selectivity to the Ni mask (i.e., ratio of $\mathrm{SiC}$ and $\mathrm{Ni}$ etch rates) was found to be reduced from 120 to 65 when platen power was increased from 50 to $100 \mathrm{~W}$, at 17-mT pressure and 17-s passivation time. Of the three parameters studied, pressure had the least significant effect on etch 
rate, probably because pressure affects etch rate through competing mechanisms. An increase in pressure reduces the plasma density, which has a negative impact on etch rate by reducing the flux of ions to the substrate. At the same time, an increase in pressure causes effects that tend to increase the etch rate; e.g., the flux of fluorine species to the substrate increases, as does the energy with which ions bombard the substrate (for a fixed platen power).

Anisotropy. Figure 2 shows the profile of trenches etched at the highest etch rate conditions (high platen power, low pressure, and low passivation time). Under these highest rate conditions, sidewalls were rougher than for samples etched using some lower rate processes. Figure 3 shows

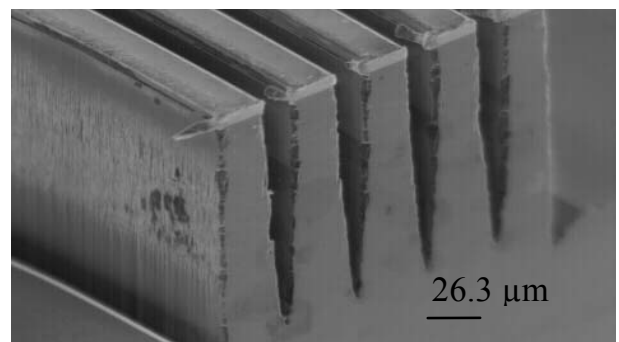

Fig. 2 - Cross-section of trenches etched with highest etch rate conditions $(100 \mathrm{~W}, 7 \mathrm{mT}, 9 \mathrm{~s})$. Trench width $=13 \mu \mathrm{m}, \mathrm{AR}=7.6$. the variation of sidewall angle as a function of pressure and platen power for a 17-s passivation step. Higher pressure and platen power during etching resulted in more vertical sidewalls, with both variables appearing to have equal effects. Increased platen power causes an increase in ion directionality, resulting in greater anisotropy of features. In the range of pressures studied, increasing pressure causes the sidewall slope to increase. However it can be expected that at a critical value, anisotropy will decrease at high pressures even with increasing platen power. Passivation time did not strongly influence sidewall angle, however, a longer passivation step did produce significantly smoother sidewalls, as shown in Fig. 4, which compares samples etched with 9-s and 17-s passivation times at high platen power and pressure $(100 \mathrm{~W}, 17 \mathrm{mT})$. At these conditions for maximum anisotropy, the slope was identical, $89.0^{\circ}$, for both passivation times.
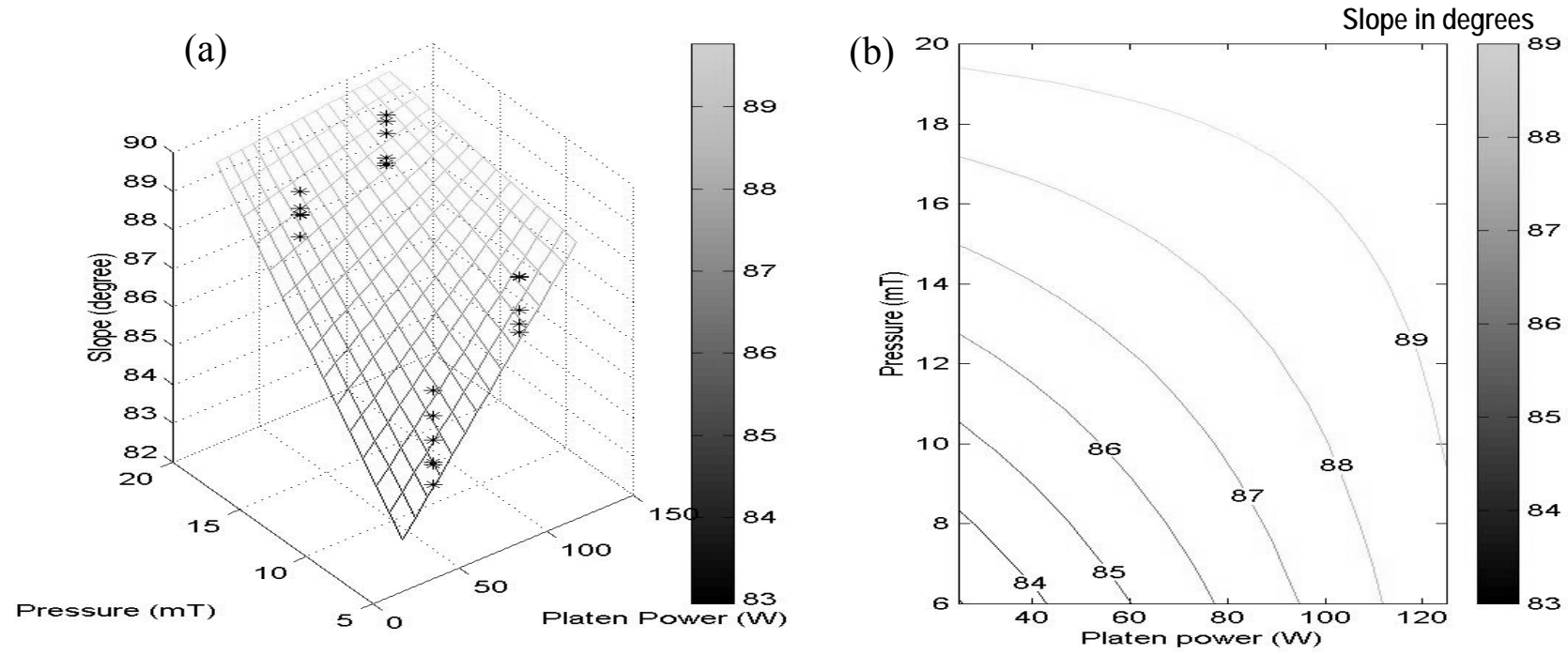

Fig. 3 - Sidewall slope as a function of platen power and pressure with a 17-s passivation time:

(a) response surface and experimental data points; (b) contour plot of response surface.

An optimized TMEP process was used to etch micro-scale turbine blades, shown in Fig. 5. High platen power, pressure and passivation time $(100 \mathrm{~W}, 17 \mathrm{mT}, 17 \mathrm{~s})$ were employed since these conditions provide maximum sidewall slope and smoothness, with an acceptable $0.17-\mu \mathrm{m} / \mathrm{min}$ etch rate. If somewhat rougher sidewalls were acceptable, then high platen power, pressure and short passivation time $(100 \mathrm{~W}, 17 \mathrm{mT}, 9 \mathrm{~s})$ could be used to obtain the same anisotropy with a higher etch rate of $0.22 \mu \mathrm{m} / \mathrm{min}$. Figure 5 illustrates some important defects with this process that need to be 
overcome by further work: (1) Higher sidewall slopes (greater anisotropy) are desired.

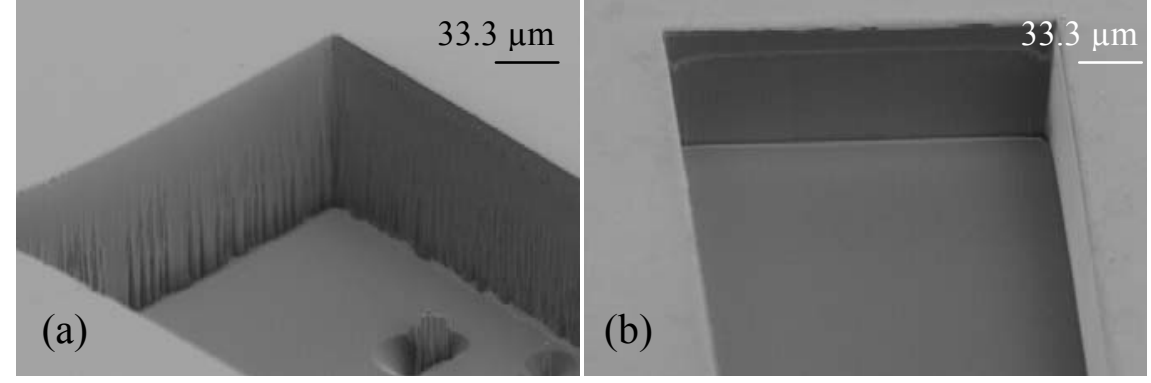

Fig. 4 - Effect of passivation time on sidewall smoothness at $100 \mathrm{~W}, 17 \mathrm{mT}$ : (a) 9-s passivation; (b) $17-\mathrm{s}$ passivation.

(2) The trenching visible at the base of the sidewall is an undesirable result typical of SiC DRIE using $\mathrm{SF}_{6}$ (high trenching was observed in all TMEP etches). (3) The process used to produce the electroplated $\mathrm{Ni}$ mask (still visible on the tops of the blades) does not accurately reproduce fine features such as the blade tips, which should be sharper.
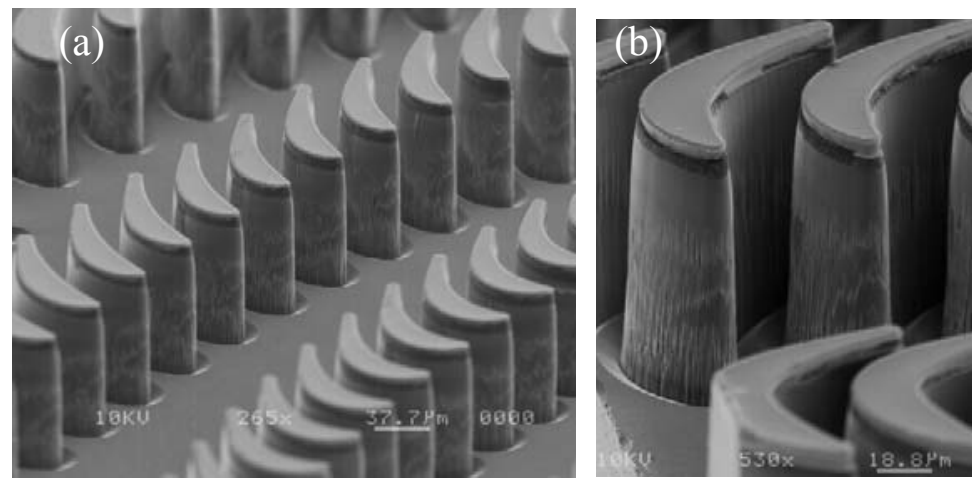

Fig. 5 - SEMs showing (a) turbine blades etched to a depth of $109 \mu \mathrm{m}$ in $6 \mathrm{H}-\mathrm{SiC}$; (b) close-up of blades, which are $24 \mu \mathrm{m}$ at the widest point and 8 $\mu \mathrm{m}$ towards the trailing edge (in background).

\section{Summary}

Design of experiments methodology was used to characterize the response dependence of $\mathrm{SiC}$ etch rate and anisotropy on etching conditions in an ICP etcher. Etch rate was primarily determined by platen power and passivation time, while sidewall slope was primarily determined by platen power and pressure. It was found that smoother sidewalls could be obtained by increasing passivation time. Independent control of etch and passivation processes allowed optimization of sidewall slope and smoothness. Aspect ratios $>5$ and etch depths $>100 \mu \mathrm{m}$ were demonstrated in microstructures of single-crystalline $\mathrm{SiC}$. With these advances in DRIE, structures can be created in $\mathrm{SiC}$ with a higher degree of control and precision than previously possible.

Acknowledgements: This work was sponsored by the Alternate Energy Foundation Technologies Subproject of the Low Emissions Alternative Power Project at the NASA Glenn Research Center. Thanks to Luc Fréchette and the Columbia University Microturbine Group for the turbine rotor mask. Thanks also to Drago Androjna, Beth Osborn, Michelle Mrdenovich, José Gonzales, Charles Blaha, James Mazor and David Spry for technical support.

\section{References}

[1] A.A. Ayon, R. Braff, C.C. Lin, H.H. Sawin, M.A. Schmidt, Journal of the Electrochemical Society, 146 (1) 339-349 (1999)

[2] F. Laermer and A. Schlip, Patent DE4241045, U.S. Patent 5,501,893 (1994)

[3] G. Beheim, in The MEMS Handbook, edited by M. Gad-el-Hak, CRC Press, Boca Raton (2002) 